\title{
C60 to CNT- CNF - Buckypaper: wonder platforms for nanomedicine applications
}

\author{
Abbreviations:CNTs, Carbon Nanotubes; CNFs, Carbon \\ Nanofibers; BP, Buckypaper; NADH, Nicotinamide Adenine \\ Dinucleotide.
}

\section{Editorial}

The name "buckypaper" is as intriguing as the awe-inspiring properties of this material. The name has its origins in the discovery by Kroto WH et al. ${ }^{1}$ which earned them the 1996 Nobel Prize for Chemistry. They had uncovered a molecule with 60 carbon atoms, unambiguously shaped like a soccer ball and as observed by Sir Kroto, with whom the author had the privilege to meet, resembled the stable geodesic domes created by architect and futurist Buckminister Fuller. They ended up naming the $\mathrm{C}_{60}$ molecule 'buckminster fullerene the other potential alternatives suggested being 'ballene, spherene, soccerene, carbosoccer'. They had rightly predicted, in their 1985 paper, the immense 'chemical and practical value' of $\mathrm{C}_{60}$ species if a large-scale synthetic route was found to make this substance.

Then came the discovery of carbon nanotubes (CNTs) by Iijima. ${ }^{2}$ of NEC Laboratories, Japan, who observed these 'needle like tubes', which are 50,000 times thinner than human hair. He proposed a new growth model and produced these structures using an arc-discharge evaporation method. During the same year (1991), a Japanese company developed a method to grow carbon nanofibers (CNFs). ${ }^{3}$ Ever since, the CNTs and CNFs, like a gold rush, have drawn much attention due to their unique chemical, mechanical, electrical and structural properties on account of the stronger $\mathrm{sp}^{2}$ bonds and as a result their applications have grown manifold. On the other hand, Buckypaper (BP) is formed by filtering CNT/CNF dispersion through a finemesh filter using suction, as accidently found by Smalley's group. Wang et $\mathrm{al}^{4}{ }^{4}$ invented a method for making BP in large sizes while enhancing the electrical (conducting current like copper/silicon) and thermal properties (dispersing heat like steel/brass) by magnetically aligning the CNTs in the paper, making them lighter than steel by 10 times while still keeping them lighter by 500 times. This obviously has shown immense potential in applications ranging from army vehicle armor, heat seeking missiles, next generation electronics, energy storage devices, fire protection, electromagnetic interference shielding and lightening-strike protection on aircraft.

Various methods of synthesis have been employed, for example, CNTs and CNFs have been fabricated using various methods like chemical vapour deposition, electric arc vaporization, laser vaporization, gas phase catalytic growth from carbon monoxide, catalytic formation, etc. ${ }^{5,6}$ On the other hand, BP has been fabricated using various coating methods (spray, spin, dip), self-assembly, drop casting, electrophoretic deposition, slot die, etc. One of the most interesting applications of these materials and which has gained much attention is in the field of nanomedicine. This has been realized due to certain features of these materials, like the high aspect ratio and the large number of side wall dangling bonds, which are very
Volume 2 Issue 3 - 2015

\author{
Annadanesh Shellikeri, ${ }^{1,2}$ \\ 'Department of Electrical and Computer Engineering, Florida \\ A\&M University, USA \\ ${ }^{2}$ Florida State University, USA
}

Correspondence: Annadanesh Shellikeri, Department of Electrical and Computer Engineering, Florida A\&M University, Levy Ave, Tallahassee, FL, USA, Tel (850) 559-2553 Email annadaneshs@gmail.com

Received: October 05, 2015 | Published: May 14, 2015

attractive in biomedical and nanomedicine applications as there is a high possibility of functionalization using numerous bioactive molecules such as peptides, proteins, nucleic acids and therapeutic drugs. Functionalization can also improve their solubility and dispensability by reducing Van der Waals forces, which is brought about by carboxyl group induced oxidation and reduce metal catalyst induced cytotoxicity, making them more biocompatible. ${ }^{6,7}$

CNTs / CNFs have come a long way ever since their discovery, taking up multiple roles and materializing into many forms. Now they have taken the role of mimicking complex structures like bones. Researchers have successfully shown that with some functionalization, the high mechanical strength and low density properties of CNTs can be utilized in bone regeneration. ${ }^{8}$ In such cases, the CNTs have been modified using negatively charged groups like amines, which make them ideal platforms to attract cations like calcium ions in human body, promoting bone growth. On the other hand CNFs have proved to be versatile material, worthy for orthopedic or dental implant, due to their high surface energy after some formulation, selectively influencing adhesion of cells, like osteoblast, fibroblast, chondrocyte, smooth muscle cell, etc. ${ }^{9}$ Moreover, the excellent properties of CNFs but at a lower cost relative to CNTs have made them a poster child of bioengineering materials. It is for this reason that they have found favour in the neural tissue engineering field. Being nano in dimensions, 3-dimensionl in structure and having high electrical conductivity, both CNFs and CNTs electrodes have been used as implants for safely and efficiently monitoring, diagnosing and stimulating repair of damaged nerves/tissues.

Wouldn't it be great if we had vehicles of transportation at the nano level which could deliver the right drug in the right amounts at the right place in human body, hence minimizing the side-effects? That is what exactly the CNTs and CNFs have been up to, which have shown the ability to cross cell membranes by endocytosis. ${ }^{10}$ as well as insertion and diffusion pathways. ${ }^{11}$ Researchers today have utilized this property and functionalized the CNTs which enable them to deliver the drug right at the place they are needed, for example a tumor, by internalization of both the drug and the carrier, which is known to have higher levels of delivery efficacy. Furthermore, the 
residual metal (Ni) catalysts in CNTs impart them with the ability to respond to an external magnetic field; hence they can be guided to enter cells using this field and has been shown to have 107-fold increase in drug delivery efficacy. Gene therapy is another field where CNFs and BP mesh have shown immense potential in delivering genes in cells, helping them promote therapeutic proteins. A biocompatible BP mesh capsule ('biocapsule') has been developed by NASA recently, which can hold living cell and tissues for transplanting into body. These methods are known to be least destructive to cell membranes than the conventional microinjection method; also allows the tracking of gene delivery and the subsequent cell proliferation processes.

Use of CNTs / CNFs in electrochemical biosensing applications has been quite extensive.$^{12}$ and this is made possible by the remarkably high sensitivity of their electrical conductivity to the surface adsorbates. By overcoming the insolubility challenge of these structures through target-specific functionalization, CNT/CNF based amperometric transducers have been developed for clinical diagnostics and environmental monitoring. For example, there are around 200 dehydrogenases and 100 oxidases, many of which generate NADH (reduced Nicotinamide adenine dinucleotide)and hydrogen peroxide, when they catalyze the reactions of certain clinically significant analytes like glucose, lactate, urate, amino acids (tryptophan, tyrosine), cholesterol, alcohol, etc, which are electrochemically detectable with minimal surface passivation of CNT based enzyme electrodes. Hence making them extremely attractive. More recently, BP has been successfully used as a support platform. ${ }^{13}$ to sense many biologically important molecules, like tryptophan, L-carnitine, tyrosine and myoglobin, showing marked increase insensitivity (1000fold) towards these molecules when compared with traditional glassy carbon electrodes. And efforts are underway to improve the specificity of these modified electrode platforms by bringing the advantages of CNTs and CNFs together and immobilizing specific enzymes.

The ever increasing applications of $\mathrm{CNTs} / \mathrm{CNF} / \mathrm{BPs}$ bring us to the question of cost. Presently, due to its application in vast variety of fields, the mass production of CNTs has become a reality, reducing the per $\mathrm{Kg}$ cost from $\$ 45000$ to $\$ 100$ while increasing the productivity to several 100 tons/year in the last 10 years of its development. ${ }^{14}$ And since the application is only going to become more diverse, the prices will continue to decline. Efforts must also be directed towards CNT/CNF based inks since these can prove to be very handy in micro fabrication of screen-printed biosensing strip electrodes. These offer an opportunity for mass production of these bio-sensitive devices, reducing costs and increasing reproducibility. The BP is also slated to go into production mode in near future with the improved manufacturing process and the development of prototype machine. ${ }^{15}$ for mass production that will only make it commercially more feasible.

Finally, a note of caution. While disagreement in the in vitro, in vivo toxicity results from different research groups regarding these innovative platforms remain, it must not be forgotten that the increased applications and the resulting mass production will result into more human exposure to these modern nanomaterials. Hence it is imperative that a more intense toxicological scrutiny of CNT/CNF/BP particles and products is to be undertaken. These are the times of great advances in nanotechnology, nanopharmacology and nanomedicine. It can be agreed upon that with the advent of new improved synthesis methods of CNTs/CNFs, better control of physical and chemical properties is possible to achieve efficient electron transfer, enhanced electroanalytical and chemical activity towards target molecules. Combining this with the mechanical, thermal and electrochemical stability of the BP electrodes makes these platforms very attractive, paving the way fora wide range of biomedical applications and next generation electrochemical biosensors.

\section{Acknowledgments}

None.

\section{Conflicts of interest}

None.

\section{References}

1. Kroto HW, Heath JR, O'Brien SC, Curl RF, Smalley RE $\mathrm{C}_{60}$ : Buckminsterfullerene. Nature. 1985;318: 162-163.

2. Iijima $\mathrm{S}$ Helical microtubules of graphitic carbon. Nature. 1991;354:56-58.

3. Morgan $\mathrm{P}$ Carbon Fibers and Their Composites. CRC Press, USA, pp.993. 2005

4. Wang D, Song P, Liu C, Wu W, Fan S Highly oriented carbon nanotube papers made of aligned carbon nanotubes. Nanotechnology. 2008;19(7):075609.

5. Dai H Carbon nanotubes: synthesis, integration, and properties. Acc Chem Res. 2002;35(12):1035-1044.

6. Tran PA, Zhang L, Webster TJ Carbon nanofibers and carbon nanotubes in regenerative medicine. Adv Drug Deliv Rev. 2009;61 (12):1097-1114.

7. Klein KL, Melechko AV, McKnight TE et al. Surface characterization and functionalization of carbon nanofibers. $J$ Appl Phys. 2008;103:061301-061326.

8. Zhao B, Hu H, Mandal SK et al. A Bone Mimic Based On The SelfAssembly Of Hydroxyapatite On Chemically Functionalized SingleWalled Carbon Nanotubes. Chem Mater. 2005;17(12):3235-3241.

9. Price RL, Waid MC, Haberstroh KM et al. Selective bone cell adhesion on formulations containing carbon nanofibers. Biomaterials. 2003;24(11):1877-1887.

10. Kam NW, O’Connell M, Wisdom JA et al. Carbon nanotubes as multifunctional biological transporters and near-infrared agents for selective cancer cell destruction. Proc Natl Acad Sci U SA. 2005;102(33):11600-11605.

11. Bianco A, Kostarelos K, Prato M Applications of carbon nanotubes in drug delivery. Curr Opin Chem Biol. 2005;9(6):674-679.

12. Joseph W Carbon-Nanotube based electrochemical biosensors: a review. Electroanalysis. 2005;17(1):7-14.

13. Cardenal JA, Shellikeri A, Chatterjee J Engineered Carbon Nanotube Buckypaper: A Platform for Electrochemical Biosensors. J Biomedical Nanotechnology. 2014;11(1):150-156(7).

14. Zhang Q, Huang JQ, Qian WZ et al. The road for nanomaterials industry: a review of carbon nanotube production, post-treatment and bulk applications for composites and energy storage. Small. 2013;9(8):1237-1265.

15. MC Anderson Buckypaper: Whatever Happened to the Aerospace Material of Tomorrow? Manufacturing Engineering magazine. 2013. 